\title{
LEPTOMONAS NAUCORIDIS N. SP., PARASITE INTESTINAL DE NAUCORIS MACULATUS FABR.
}

\author{
Par Raymond POISSON \\ Chef des travaux de zoologie à la Faculté des sciences de Caen
}

On sait que les Leptomonas, trypanosomides sans membrane ondulante, existent à l'état de parasites intestinaux, rarement colomiques, chez beaucoup d'insectes et en particulier chez les hémiptères suceurs de sang ou de sucs végétaux. Il en a été observé également chez quelques vertébrés, chez un mollusque gastéropode (A. Porter, 1914), chez des nématodes (Saville Kent, E. Chatton, 1924), et aussi dans le latex de certains végétaux (F. Mesnil, 1921).

$\mathrm{Si}$ on envisage plus spécialement les hémiptères, on constate que bon nombre d'espèces aquatiques sont parasitées par des trypanosomides. C'est ainsi que l'on connaît chez ces dernières : Leptomonas jaculum Léger, de Nepa cinerea L. (L. Léger, 1902; A. Porter, 1910, etc.) ; Crithidia gerridis Patton, parasite intestinal chez Gerris fossarum Fabr., G. paludum Fabr., G. remigis Say., G. marginatus Say., G. rufoscutellatus Latr., Microvelia americana Uhl. (W.-S. Patton, 1908 ; A. Porter, 1910 ; E.-R. Becker, 1923, etc.) ; Leptomonas gerridis chez des Gerris (1) (J.-S. Dunkerly, 1913) ; L. veliæ et Crithidia veliæ chez Velia currens Fabr. (J.-S. Dunkerly, 1913).

Cette rapide revue d'ensemble des espèces d'hémiptères aquatiques parasitées permet de supposer qu'il n'a pas encore été observé de trypanosomides chez les Naucoridæ, Notonectidæx et Corixidæ. Or, au cours de mes recherches sur ces insectes, j'ai pu constater à maintes reprises la présence de Leptomonas intestinaux chez diverses espèces appartenant aux familles précédentes.

Je décrirai dans cette note un Leptomonas parasite de Naucoris maculatus Fabr. qui, à ma connaissance, n'a pas encore été étudié et que je nomme Leptomonas naucoridis n. sp.

Provenance des naucores parasitées. - Naucoris maculatus Fabr. est un hétéroptère assez commun, et même parfois abondant, dans

(1) Je n'ai pu me procurer le mémoire original et ne sais si l'auteur a précisé quelles sont les espèces parasitées par ce flagellé.

Annales de Parasitologie, $\mathrm{T}$. III, $\mathrm{N}^{\circ}$ 1. - Janvier 1925, p. 28-34. 
les eaux saumâtres supralittorales normandes. Il est rare au contraire dans les eaux douces de l'intérieur des terres. Les exemplaires parasités, dont je me suis servi pour l'étude du flagellé, proviennent tous de mares ou ruisseaux d'eau saumâtre, dont la teneur en chlorure de sodium varie entre 1 gr. et 7 gr. par litre. En mai 1924, toutes les naucores examinées étaient abondamment parasitées. Par contre, les individus capturés en eau douce sont rarement parasités et quand ils renferment des flagellés ces derniers sont rares.

N. maculatus vit dans les stations saumâtres littorales en compagnie d'autres hémiptères. J'y ai capturé, entre autres, des nèpes non parasitées en général par L. jaculum; Microvelia pygmæa non parasitée par Crithidia gerridis; un certain nombre d'espèces de Gerris dont beaucoup d'exemplaires renferment des Crithidia (C. gerridis) ; enfin des notonectes et des corises qui hébergent parfois aussi des Leptomonas. $N$. viridis Delc. est, en particulier, souvent infestée.

Description de Leptomonas naucoridis n. sp. - Chez les naucores adultes, provenant des stations précédemment mentionnées, les formes monadiennes du parasite sont souvent très abondantes dans tout l'intestin moyen. Ces formes se présentent comme des organismes aciculés dont les plus grands exemplaires (fig. 1, a) mesurent $25 \mu$ de longueur sur 2 à $2 \mu, 5$ de largeur. Mais les individus les plus nombreux mesurent seulement $15 \mu$. La longueur du flagellum, porté par le pôle antérieur du parasite, atteint le plus souvent le double de celle du corps.

Parmi ces formes allongées, certaines sont grêles; d'autres, au contraire, ont un aspect de massue, renflées qu'elles sont dans leur région antérieure (fig. $1, c$ ).

Au stade monadien le parasite, en se fixant par son flagellum sur des particules alimentaires, constitue des rosaces d'agglomération abondantes en individus (fig. $1, d$ ).

Sur coupe on remarque que beaucoup de flagellés sont fixés directement à l'épithélium intestinal par leur fouet considérablement raccourci (fig. $1, e$ ). Ce sont là des formes grégariniennes (L. Léger, 1902) ; ces formes sont d'autant plus abondantes que l'imago de naucore est plus âgée.

Après fixation et coloration convenables (Giemsa ; Schaudinnhématoxyline au fer, etc.), on peut distinguer, à l'intérieur de l'endoplasme, un noyau ovoïde à gros karyosome situé, le plus souvent, dans la région antérieure du parasite. Au-dessus du noyau, et assez près de la surface du corps, on remarque la présence d'un élément sidérophile légèrement allongé : c'est le blépharoplaste. De 
ce dernier se détache la portion radiculaire du flagellum ; quelques rares individus, parmi les petites formes, peuvent présenter un rhizoplaste nucléaire.

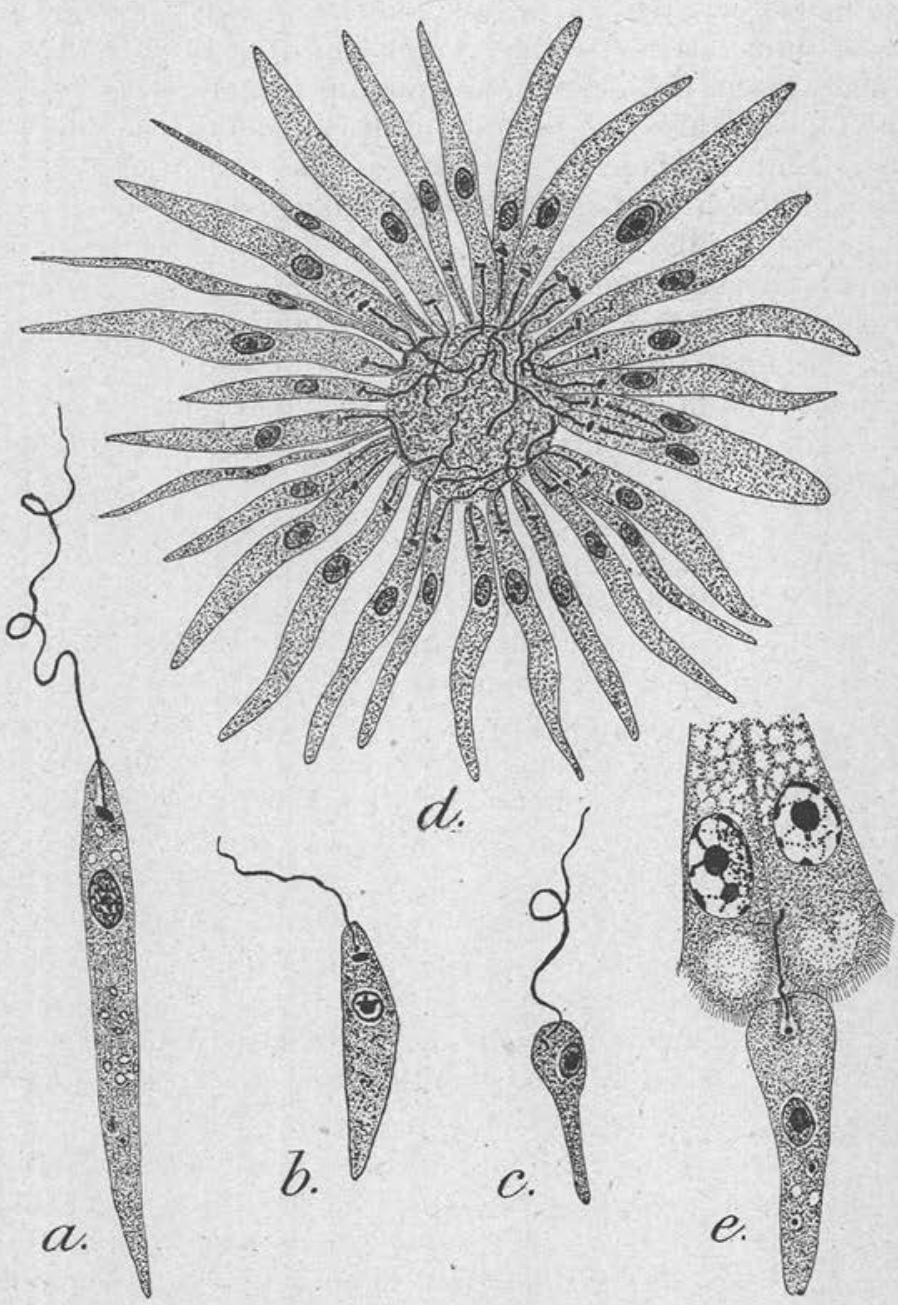

FIG. 1. - Leptomonas naucoridis n. sp. $\times 3000 .-a$, grande forme aciculée ; $b$, forme moyenne ; $c$, forme en massue ; $d$, rosace d'agglomération ; $e$, forme grégariuienne fixée à l'épithélium intestinal.

Enfin je signalerai encore l'existence de myonèmes, situés sous un mince périplaste (fig. $1, a$ ).

Je n'ai pas observé de v́acuole buccale ni de canal alimentaire 
rappelant les formations que L. Léger (1902) a décrites chez L. jaculum (1) ; mais, une solution très diluée de rouge neutre (1 p. 20.000) colore vitalement le contenu de 2 ou 3 vacuoles de moyenne taille situées dans la région antérieure, de part et d'autre de la base du flagelle, ou un peu en-dessous de cette base ; il existe aussi quelques petites vacuoles postérieures plus petites.

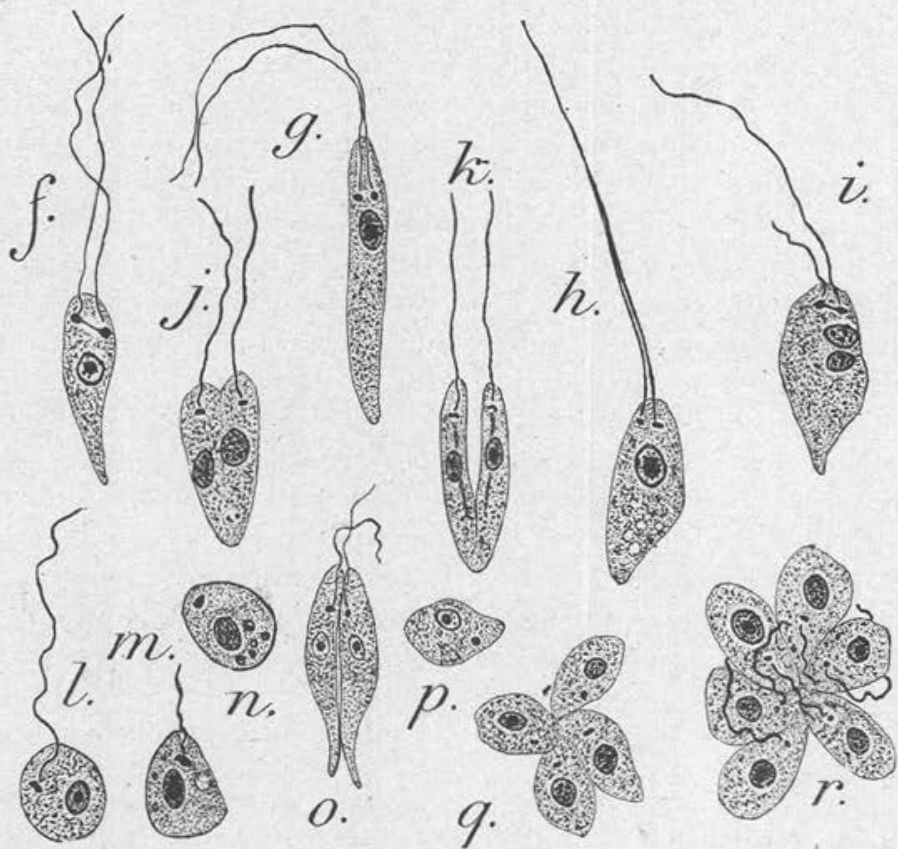

FIG, 2. - Leptomonas naucoridis n. sp. $\times 3000,-f$ à $k$, division longitudinale du flagellé; $l, m$, formes arrondies de l'intestin postérieur (prélude à l'enkystement); $o$, conjugaison (?) de deux formes monadiennes; $p$, forme leishmanienne de l'intestin moyen antérieur; $q$, rosace de multiplication de formes leishmaniennes ; $r$, rosace de multiplication de formes flagellées.

Multiplication du parasite (fig. 2, $f$ à $k$ ). - Les formes monadiennes se multiplient activement dans tout l'intestin moyen des naucores adultes, par division longitudinale ; mais ce sont surtout les formes de taille moyenne, trapues, que j'ai observées le plus fréquemment en voie de division.

Les processus de la division longitudinale des Leptomonas sont connus. Je signalerai seulement, chez $L$. naucoridis, la persistance fréquente d'un axoplaste entre les deux blépharoplastes fils et le

(1) Ces deux formations n'ont pas été revues par A. Porter (1910). 
fait que le flagellum paraît ou bien se dédoubler dans toute sa longueur (fig. $2, h$ ) ou bien se former à partir du nouveau blépharoplaste (fig. $2, i$ ). Mais ce dernier mode parait peu fréquent.

J'ai obtenu de nombreuses divisions du parasite en plaçant des intestins dans la solution de Ringer.

Enkystement du parasite. - Les formes monadiennes, qui passent dans l'intestin postérieur et dans les ampoules rectales (1) de la naucore, ne conservent pas leur aspect aciculaire. D'allongées, elles deviennent plus ou moins arrondies et leur flagelle se raccourcit (fig. $2, l, m$ ), puis bientôt disparaît (fig. $2, n$ ). Après fixation au liquide de Zenker acétifié, ou encore au Duboseq-Brasil, suivie de la coloration trichromique de Prenant, on remarque que ces parasites rectaux aflagellés présentent une mince gaine protectrice éosinophile (cf. E. Chatton, 1913). Le noyau prend un aspect pyenotique et de la chromatine est rejetée sous forme de granules dans le cytoplasme (fig. $2, n$ ). Ces kystes mesurent de 4 à $7 \mu$ de diamètre ; ils sont rejetés dans le milieu extérieur et représentent vraisemblablement les éléments de propagation du parasite.

Je n'ai pas observé dans les préliminaires à l'enkystement de phase de copulation entre formes flagellées, rappelant, entre autres, ce que S. Prowazek (1904) a décrit chez L. muscæ-domesticæ Werner et L. sarcophagæ Prow. Cependant j'ai remarqué dans des intestins parasités, conservés dans la solution physiologique, des accolements deux à deux de formes monadiennes (fig. $2, o$ ) qui précèdent peut-être un enkystement double ; mais je n'ai pu assister jusqu'à présent à la suite de ce processus.

Début de l'infection. - J'ai observé des cas d'infection naissante chez des larves de $N$. maculatus parvenues au quatrième stade larvaire. On peut alors trouver dans la portion antérieure de l'intestin moyen et entre les villosités intestinales, des formes aflagellées, peut-être amiboïdes (fig. $2, p$ ), présentant un centrosome et un noyau avee gros caryosome. Ces individus leishmaniens sont susceptibles de se multiplier tout en restant aflagellés (fig. 2, q). Mais, à côté de ces multiplications de formes sans flagelles qui sont rares, on observe aussi des rosaces dont les individus présentent chacun un court et gros flagellum (fig. $2, r$ ). Tous les individus de ces rosaces convergent par leur pôle antérieur. Ces jeunes formes monadiennes mesurent 6 à $8 \mu$ de longueur sur $2 \mu, 5$ à $3 \mu$ de largeur. Elles se multiplient activement par division longitudinale, une fois les rosaces dissociées, si bien que chez les larves âgées l'intestin moyen est déjà abondamment parasité.

(1) Il existe, chez $N$. maculatus, deux ampoules rectales. 
Les formes aflagellées leishmaniennes de l'intestin moyen sont très rares chez les naucores adultes.

Formes cœlomiques. - Leptomonas naucoridis est le plus généralement localisé au tube digestif. Mais dans des cas d'infection particulièrement intense j'ai observé de nombreuses formes cœlomiques (1). Celles-ci mesurent de 7 à $10 \mu$ de longueur et sont par conséquent plus petites que les formes intestinales; en outre, leur flagelle est plus épais.

Les formes cœlomiques sont susceptibles de pénétrer dans certains organes. Je signalerai à cet égard en avoir observé à plusieurs reprises dans les glandes labiales et, dans ce cas, les parasites sont surtout localisés à la glande accessoire (2). Comme les naucores sont des insectes carnassiers s'attaquant parfois aux jeunes poissons et aux batraciens, il est possible qu'elles puissent inoculer le trypanosomide à certains de ces vertébrés aquatiques. Des recherches en cours me permettront peut-être d'élucider ce point.

J'ai aussi constaté chez deux naucores, présentant une infection cœlomique massive, la présence de formes monadiennes du flagellé dans une petite glande ventrale métathoracique (3).

Enfin, je mentionnerai encore avoir remarqué l'existence de stades leishmaniformes dans l'épithélium ovarique et parfois dans les jeunes ovules. Mais comme je n'ai jamais trouvé d'embryoñs parasités, j'en déduis que ces formes aflagellées dégénèrent. Cette observation est à mettre en parallèle avec celle que A. Porter (1910) a faite sur $L$. jaculum. L'auteur déclare, en effet, avoir trouvé des formes leishmaniennes du parasite dans les ovaires des nèpes, mais ces formes dégénèrent au cours du développement.

\section{RÉSUMÉ}

Les individus de $N$. maculatus capturés dans les eaux saumâtres supralittorales normandes sont le plus souvent abondamment parasités par un Leptomonas que je nomme Leptomonas naucoridis

(1) ZoттA (1912) a fait une semblable constatation en ce qui concerne le Leptomonas parasite du Pyrrhocoris apterus L. L'on sait, d'autre part, qu'il existe des cas où les Leptomonas sont uniquement cœlomiques.

(2) Les glandes labiales ou salivaires de la naucore sont paires. Il existe, de part et d'autre de l'intestin antérieur, une glande principale bilobée et une glande accessoire, ou réservoir, réunie à la première par un canal (voir R. Poisson, 1924).

(3) Cette glande, dont l'existence m'avait échappé dans mes recherches antérieures, s'ouvre par deux ostioles situés au fond de deux enfoncements, de part et d'autre d'une carène métasternale s'étendant entre les pattes postérieures. Les coxas de ces pattes cachent normalement les ostioles. Il existe chez la larve des glandes dorso-abdominales rudimentaires dont les orifices excréteurs, situés de part et d'autre de la ligne médio-dorsale à la limite du $3^{e}$ tergite, sont difficiles à voir. 
n. sp. Ce flagellé, généralement localisé au tube digestif, peut, dans les cas d’infection massive, envahir la cavité générale et certains organes, les glandes salivaires par exemple.

De même que chez les autres Leptomonas parasites intestinaux d'insectes, les divers stades de l'évolution de $L$. naucoridis, chez la naucore, sont localisés à certaines régions du tube digestif. Dans la région antérieure de l'intestin moyen on observe des formes aflagellées, isolées ou en rosaces de multiplication ainsi que des rosaces de multiplication de formes flagellées.

Dans l'intestin moyen on trouve des formes monadiennes aciculaires, se multipliant activement par division longitudinale ; des formes grégariniennes fixées aux cellules de l'épithélium intestinal ; des rosaces d'agglomération et aussi des couples de formes flagellées aciculaires représentant peut-être une copulation (?) précédant l'enkystement.

Enfin dans l'intestin postérieur et les ampoules rectales, il existe des formes flagellées arrondies, dont le fouet est en voie de régression, et des kystes.

\section{BiBLIOGRAPHIE}

Becker (E.-R.). - Observations on the morphology and life-cycle of Crithidia gerridis Patton in the water striders Gerris remigis Say. Journ. of parasitology, IX, 1923, p. 141-152.

Chatron (E.). - L'ordre, la succession et l'importance relative des stades dans l'évolution des trypanosomides chez les insectes. C. R. Soc. Biol., LXXIV, 1913, p. 1145.

- Sur un Leptomonas d'un nématode marin et la question de l'origine des trypanosomides. C. R. Soc. Biol., XC, 1924, p. 780.

DunKerLy (J.-S.). - On some stages in the life history of Leptomonas muscadomestice with some remarks on the relationships of the flagellate parasites of Insects. Quart. journ. of microsc. Sc., LVI, 1911, p. 645.

- Flagellata and Ciliata in Clare Island survey. Proc. Roy. Irish. Acad., XXXI. Analyse in Bull. Inst. Past., 1913, p. 628.

Mesnil (F.). - La "flagellose " ou " leptomoniase » des Euphorbes et des Asclépiadacées. Ann. Sc. nat. bot., (10), III, 1921.

Patton (W.-S.). - The life-cycle of a species of Crithidia parasitic in the intestinal tract of Gerris fossarum Fabr. Arch. für. Protist., XII, 1908, p. 131.

Porter (A.). - The life-cycle of $H$. jaculum Léger parasitic in the alimentary tract of $N$. cinerea. Parasitotogy, II, 1910, p. 367.

- The morphology and life-history of Crithidia gerridis Patton as found in the British water-bug Gerris paludum. Parasitology, II, 1910, p. 348.

Prowazek (S.). - Die Entwicklung von Herpetomonas, einem mit den Trypanosomen verwandten Flagellaten. Arb. Kais. Gesund., XX, 1904, p. 440-452.

Poisson (R.). - Contribution à l'étude des Hémiptères aquatiques. Bull. Biol. France et Belg., LVIII, 1924, p. 49-305.

Zotra (G.). - Sur un flagellé du type Herpetomonas chez Pyrrhocoris apterus. Ann. Scient. Univ. Jassy, VII, 1912, p. 210-223. 